\title{
Improvement of thermal properties and flame retardancy of epoxy-amine thermosets by introducing bisphenol containing azomethine moiety
}

\author{
X. H. Zhang ${ }^{*}$, L. H. Huang ${ }^{2}$, S. Chen ${ }^{3}$, G. R. $Q i^{1}$ \\ ${ }^{1}$ Department of Polymer Science and Engineering, Zhejiang University, Hangzhou, 310027, P. R. China \\ ${ }^{2}$ Department of Applied Biology, Shenzhen Senior Technical Institute, 518048, P. R. China \\ ${ }^{3}$ College of Chemistry and Chemical Engineering, Jishou University, Jishou, 416000, P. R. China
}

Received 18 March 2007; accepted in revised form 2 May 2007

\begin{abstract}
A novel bisphenol 1, 4'-bis\{4-[(4-hydroxy) phenyliminomethylidene] phenoxy\} benzene (BHPB), which contains azomethine moiety and flexible aromatic ether linkage, was synthesized and introduced into the curing system composed of diglycidyl ether of bisphenol A (DGEBA) and diamine. The curing behavior of DGEBA/diamine changed dramatically due to the introduction of BHPB. The resultant epoxy thermosets containing BHPB had high $T_{g} \mathrm{~s}$ $\left(127-160^{\circ} \mathrm{C}\right)$, high $T_{d, 5 \%}\left(\geq 330^{\circ} \mathrm{C}\right)$ and high integral procedure decomposition temperature (IPDT) values $\left(662-1230^{\circ} \mathrm{C}\right)$ and good flame retardancy because of their high Limiting Oxygen Index (LOI) values (above 29.5).
\end{abstract}

Keywords: thermosetting resins, thermal stability, flame retardancy, epoxy, bisphenol

\section{Introduction}

Diglycidyl ether of biphenyl A (DGEBA) is widely used for its versatile properties and low cost. However, it is thermally unstable and flammable when used in insulation materials. Introducing phosphorus element $[1,2]$, which is more environmental friendly than halogens, into the main chain of DGEBA via chain extension reaction is a good way to improve the flame retardancy of the resultant thermoset. But the higher the phosphorus content, the lower the thermal stability [2-4]. Nitrogen-containing epoxy thermosets are gradually attracting more attention because they exhibit good flame retardancy, thermal properties, as well as more environmental friendly.

It is well known that bisphenols containing azomethine group $(\mathrm{Ar}-\mathrm{CH}=\mathrm{N}-\mathrm{Ar})$, which is a rigid structure, frequently used as the mesogenic unit for liquid-crystalline epoxy themosets (LCTs) [5-7], can strengthen the thermal stability and increase the char residue when the thermosets containing of them decompose. However, the yield of azomethine-containing epoxy monomers from bisphenols is very low [7], which limited their applications. Furthermore, rigid azomethine moiety may result in brittleness of the resultant thermosets if no flexible structures were introduced. Herein, we synthesized a bisphenol, 1, 4'-bis\{4-[(4-hydroxy) phenyliminomethylidene] phenoxy benzene (BHPB), which contains both the azomethine moiety and flexible aromatic ether linkage, and introduced it directly into the backbone of epoxy-amine thermosets as a 'modifier' to improve the thermal stability and flame retardancy. 


\section{Materials and methods}

Hydroquinone, $p$-chloronitrobenzene, 4-hydroxybenzaldehyde, hydrazine hydrate $(85 \mathrm{wt} \%$ water solution), potassium carbonate and Iron (III) chloride $\left(\mathrm{FeCl}_{3} \cdot 6 \mathrm{H}_{2} \mathrm{O}\right)$ and $\mathrm{ZnCl}_{2}$ were purchased from Sinopharm Chemical Reagent Co., Ltd. (Shanghai, China) and used without purification. Ethanol, dimethylformamide (DMF) were purchased from Hangzhou Shuanglin Chemical Reagent Co., Ltd. (Hangzhou, China), ethanol was used as received, and DMF was purified by distillation under reduced pressure over calcium hydride before use. Diglycidyl ether of biphenyl A (DGEBA, NPEL127E) was granted kindly from Nan Ya plastics of China with an equivalent epoxy molecular weight of $180 \mathrm{~g} / \mathrm{eq}$.

Infrared spectra were recorded by using a Vector 22 FTIR spectrophotometer (400-4000 $\mathrm{cm}^{-1}$, KBr pellet). ${ }^{1} \mathrm{H}$ NMR and ${ }^{13} \mathrm{C}$ NMR were obtained with an Avance DMX500 NMR spectrometer (Bruker, Darmstadt, Germany) at $35^{\circ} \mathrm{C}$ using $d_{6}$-DMSO as a solvent. Elemental analysis was performed on a Flash EA1112 CHN-O-Rapid elemental analyzer using acetanilide as a standard. Curing behavior of BAPB/BHPB/DGEBA system was carried out on a Perkin-Elmer DSC 7 thermal analyzer at a heating rate of $10^{\circ} \mathrm{C} / \mathrm{min}$ at a flow rate of $40 \mathrm{ml} / \mathrm{min}$ under $\mathrm{N}_{2}$. The temperature calculation was regularly made using In and $\mathrm{Zn}$ standards at each scanning rate being used. $T_{g}$ determination was carried out in DSC at a heating rate of $20^{\circ} \mathrm{C} / \mathrm{min}$ under $\mathrm{N}_{2}$. Thermogravimetric analyses (TGA) were carried out on a Perkin-Elmer Pyris 1 at a heating rate of $10^{\circ} \mathrm{C} / \mathrm{min}$ from $50^{\circ} \mathrm{C}$ to $850^{\circ} \mathrm{C}$ under $\mathrm{N}_{2}$ atmosphere. $L O I$ values were measured on a HC-2 LOI tester according to ASTM D-2863-77. The percentage in the $\mathrm{O}_{2}-\mathrm{N}_{2}$ mixture deemed sufficient to sustain the flame was taken as the $L O I$.

\section{Experimental}

\subsection{Synthesis}

The synthesis of 1, 4'-bis\{4-[(4-hydroxy) phenyliminomethylidene] phenoxy benzene (BHPB) is shown in Figure 1.

1, 4'-bis(4-nitrophenoxy) benzene, [BNPB, Figure $1,(1)$ ] was synthesized by the reaction of hydroquinone $(0.10 \mathrm{~mol})$ and p-chloronitrobenzene $(0.22 \mathrm{~mol})$ in DMF $(150 \mathrm{ml})$ in the presence of potassium carbonate $(0.22 \mathrm{~mol})$. The mixture was heated to $145-150^{\circ} \mathrm{C}$ under $\mathrm{N}_{2}$ atmosphere, and then kept about 8-10 h. After cooled to the room temperature, the mixture was poured into the mixture of water/ethanol $(1 / 1, \mathrm{v} / \mathrm{v})$ and filtered to give solid product and then recrystallized from ethanol. Yield: $92 \%$.

BNPB $(0.10 \mathrm{~mol}), 1.50 \mathrm{~g} \mathrm{FeCl}_{3} \cdot 6 \mathrm{H}_{2} \mathrm{O}, 1.50 \mathrm{~g}$ active carbon and $300 \mathrm{ml}$ ethanol were introduced into a $1000 \mathrm{ml}$ three-necked flask to which hydrazine hydrate $(85 \mathrm{wt} \%, 60 \mathrm{ml})$ was added dropwise over a period of $1 \mathrm{~h}$ at reflux temperature, after the addition was complete, the reaction continued for about $5 \mathrm{~h}$. The mixture was then filtered to remove the catalysts, after cooled to the room temperature; the product [Figure 1, (2)] was precipitated from the filter to obtain 1, 4'-bis(4-aminephenoxy) benzene (BAPB). Yield: $95 \%$.

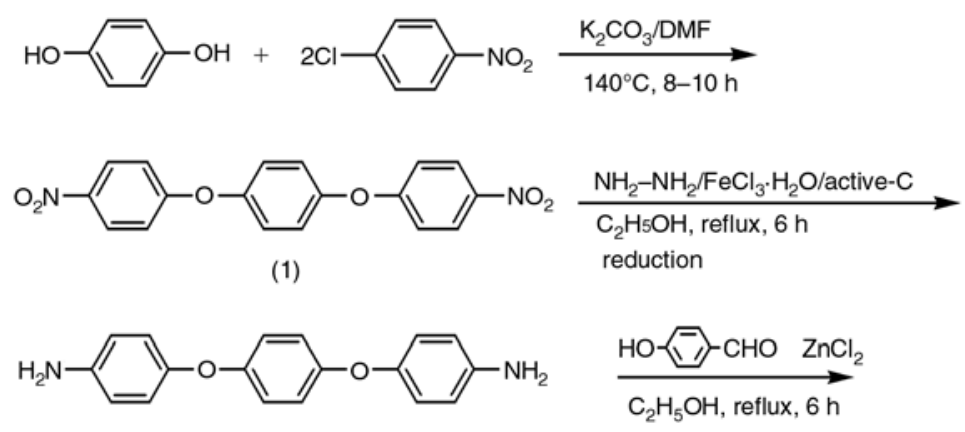

(2)

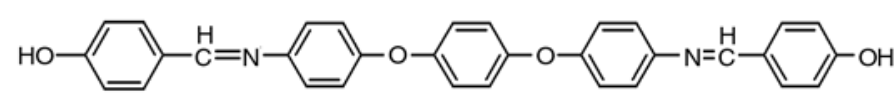

(3)

Figure 1. Synthesis routes of bisphenol BHPB. (1) 1, 4'-bis(4-nitrophenoxy)benzene (BNPB); (2) 1, 4'-bis(4-aminephenoxy)benzene (BAPB); (3) 1, 4'-bis \{4-[(4-hydroxy)phenyliminomethylidene]phenoxy $\}$ benzene BHPB) 
BAPB (0.10 mol), 4-hydroxybenzaldehyde $(0.20 \mathrm{~mol})$ and a catalytic amount of $\mathrm{ZnCl}_{2}$ were dissolved in $300 \mathrm{ml}$ of dry ethanol. The reaction mixture was stirred at reflux temperature for $6 \mathrm{~h}$, and then was poured into water/ethanol $(1 / 1, \mathrm{v} / \mathrm{v})$. The product [BHPP, Figure 1, (3)] was collected by filtration and washed by deionized water and ethanol thoroughly. Yield: $97 \%$, m.p. $278-280^{\circ} \mathrm{C}$. BHPB: IR $\left(\mathrm{KBr}, \mathrm{cm}^{-1}\right)$ : $3368(\mathrm{Ar}-\mathrm{OH}), 1605$ $(\mathrm{HC}=\mathrm{N}), 1245(\mathrm{Ar}-\mathrm{O}-\mathrm{Ar}) .{ }^{1} \mathrm{H}$ NMR $(500 \mathrm{MHz}$, ppm, DMSO- $\left.d_{6}\right): 10.10(-\mathrm{OH}), 8.47(\mathrm{CH}=\mathrm{N}), 7.76$, $7.78(\mathrm{~d}, 4 \mathrm{H}), 7.26,7.27(\mathrm{~d}, 4 \mathrm{H}), 7.01,7.08(\mathrm{~m} 8 \mathrm{H})$, 6.87-6.89 (d, 4H). ${ }^{13} \mathrm{C}$ NMR $(500 \mathrm{MHz}, \mathrm{ppm}$, DMSO- $\left.d_{6}\right)$ : $116.20,119.38,110.16,122.96$, 128.08, 130.97, 147.81, 152.92, 155.41, 159.82, $161.06(\mathrm{C}=\mathrm{N})$. Elemental Analysis: $\mathrm{C}_{32} \mathrm{H}_{24} \mathrm{~N}_{2} \mathrm{O}_{4}$ (500.55): Cal: C, 76.79\%; H, 4.86\%, N, 5.60\%; Found: C, 76.64\%; H, 4.98\%, N, 5.63\%. (The IR, NMR and Elemental Analysis data of BNPA and BAPB were omitted here for the sake of brevity.)

\subsection{Preparation of epoxy thermosets}

BAPB was mixed with DGEBA at an equivalent molar ratio, as BHPB could not dissolve into DGEBA very well. The hybrid curing agents BHPB/BAPB were prepared in various molar ratios $(2,1.5,0.3$ and 0.125 , and denoted as B1, B2, B3 and $\mathrm{B} 4$, respectively), and then mixed with DGEBA at an equivalent molar ratio in DMF (weight ratio of sample/DMF is about 1:1) under $60^{\circ} \mathrm{C}$, and then the mixture was vacuum dried at ambient temperature to obtain ternary systems. These samples were kept in the refrigerator $\left(-10^{\circ} \mathrm{C}\right)$ for DSC test.

For preparing epoxy thermosets containing BHPB for $L O I$ test, prepolymerization of samples is necessary. The procedure is as following: the sample was dissolved in a small quantity of DMF (weight ratio of sample/DMF is about $1: 1$ ), heated to $120^{\circ} \mathrm{C}$ under stirring. With the evaporation of DMF, the transparent sample was obtained. The thermosets for TGA measurement were prepared by curing the samples at $160^{\circ} \mathrm{C}$ for $1 \mathrm{~h}$ and $180^{\circ} \mathrm{C}$ for $2 \mathrm{~h}$. Specimens for $L O I$ test were obtained from the prepreg which needs pre-curing process $\left(160^{\circ} \mathrm{C} / 10 \mathrm{~min}\right)$, and then heat curing in a heat-press with the procedure of $130-160^{\circ} \mathrm{C} / 0.5 \mathrm{~h}$ and $180^{\circ} \mathrm{C} / 2 \mathrm{~h}$ for complete curing of the samples.

\section{Results and Discussion}

\subsection{Synthesis}

A novel bisphenol, 1, 4'-bis \{4-[(4-hydroxy) phenyliminomethylidene] phenoxy $\}$ benzene (BHPB) containing rigid azomethine moiety $(\mathrm{Ar}-\mathrm{CH}=\mathrm{N}-\mathrm{Ar})$ and flexible aromatic ether linkage (Ar-O-Ar), was synthesized by the nucleophilic addition reaction of BAPB [Figure 1, (2)] with 4hydroxybenzaldehyde in the presence of $\mathrm{ZnCl}_{2}$ respectively. Figure 2 shows the ${ }^{1} \mathrm{H}$ NMR and ${ }^{13} \mathrm{C}$ NMR spectra of BHPB respectively (The IR and MS spectrum of BHPB is omitted for brevity). The chemical shifts of all protons and carbons have been labeled in Figure 2 clearly. The proton of $\mathrm{CH}=\mathrm{N}$ in ${ }^{1} \mathrm{H} \mathrm{NMR}$ is at $8.47 \mathrm{ppm}$, the chemical shift of Ar-OH shifts to lower field and is at $10.10 \mathrm{ppm}$ due to introduction of azomethine moiety, and the carbon of $\mathrm{CH}=\mathrm{N}$ in ${ }^{13} \mathrm{C} \mathrm{NMR}$ is in $161.06 \mathrm{ppm}$. In the next, BHPB was used as a modifier for the BAPB/DGEBA system for improvement of the heat- and flame-resistance.

\subsection{Curing behavior}

The curing behavior of the ternary curing system containing BAPB, BHPB and DGEBA was studied by dynamic DSC method. As shown in Figure 3 curve $a$, the temperature at which the maximum conversion rate occurs in the DSC curve $\left(T_{p}\right)$ of the BHPB/DGEBA system is about $213^{\circ} \mathrm{C}$ and higher than that of BAPB/DGEBA system (curve $f$, $184^{\circ} \mathrm{C}$ ). This indicates that BHPB is difficult to react with oxirane group compared with BAPB. A small endothermic peak at about $275^{\circ} \mathrm{C}$ in curve $a$ is found which is close to the melting point of BHPB. Moreover, curve $b$ (the molar ratio of $\mathrm{BHPB} / \mathrm{BAPB}$ is 2) exhibits no significant change compared with curve $a$. These indicate that more BHPB in the curing system may be not favorable for complete curing. When the molar ratios of $\mathrm{BHPB} / \mathrm{BAPB}$ are 1.5 and 0.33 (curve $c$ and $d$ ), two obvious exothermal peaks are found in their curves and the area of the first peak increases and their $T_{p} \mathrm{~s}$ shift to higher temperature with increasing molar content of BAPB, while the endothermic peak around $275^{\circ} \mathrm{C}$ becomes smaller gradually, and no endothermic peak is found when the molar ratio of $\mathrm{BHPB} / \mathrm{BAPB}$ is 0.125 (curve $e$ ). Although curves $d$ and $e$ are unsymmetrical, it can be seen that their $T_{p} \mathrm{~s}$ are $154^{\circ} \mathrm{C}$ and $162^{\circ} \mathrm{C}$ for B3 and B4/DGEBA 

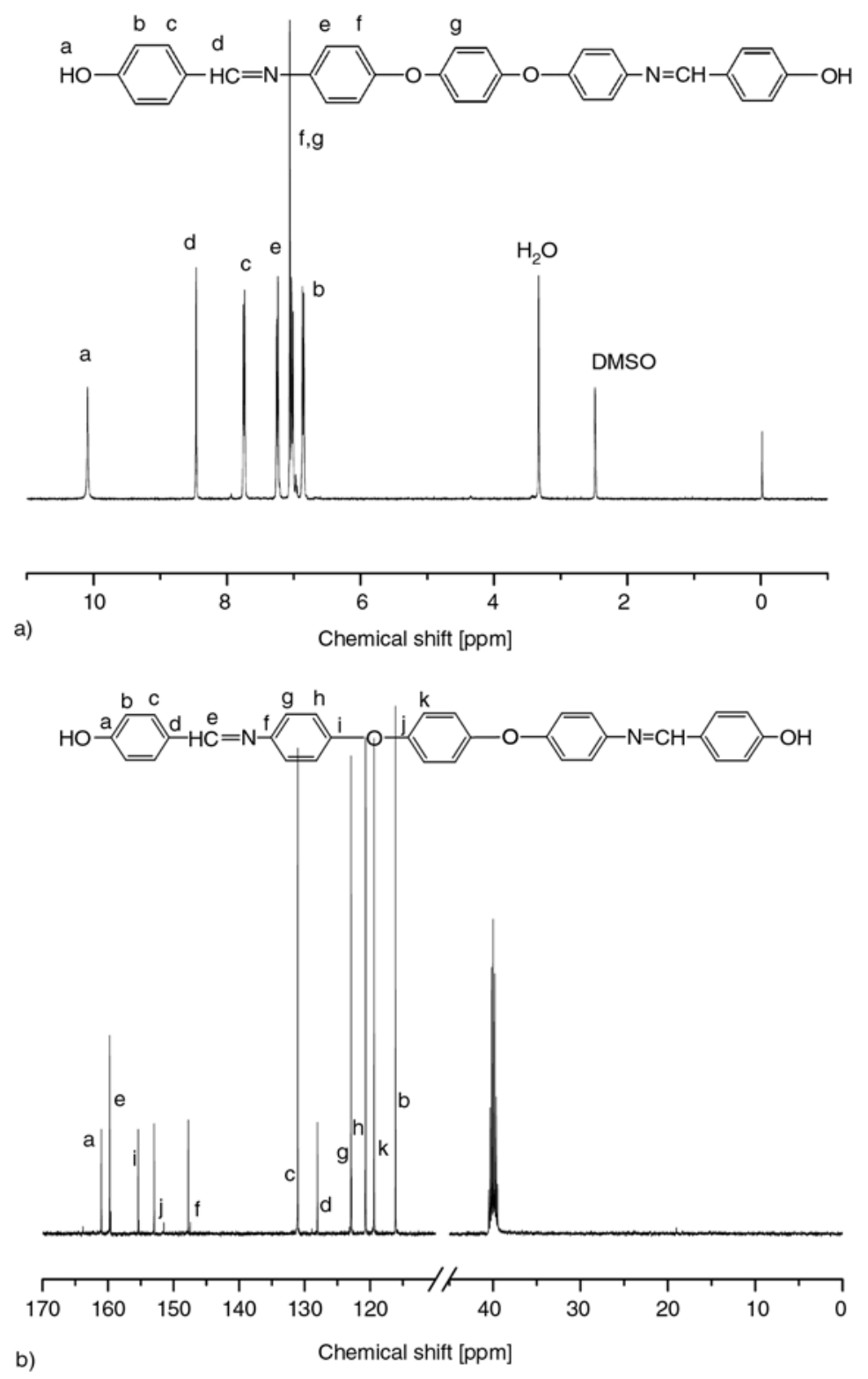

Figure 2. ${ }^{1} \mathrm{H}$ NMR (a) and ${ }^{13} \mathrm{C}$ NMR (b) spectra of BHPB

system respectively, which are obviously lower than those of BHPB/DGEBA system $\left(213^{\circ} \mathrm{C}\right)$ and BAPB/DGEBA system $\left(184^{\circ} \mathrm{C}\right)$. Thus, introduction of the suitable amounts of BHPB (BHPB/ BAPB is about less than 0.33) into the BAPB/ DGEBA system made the curing reaction of BAPB and DGEBA easier to occur.

\subsection{Thermal stability and flame retardancy}

The glass transition temperatures $\left(T_{g} \mathrm{~s}\right)$ of all epoxy thermosets were measured by a second heating trace with DSC (Figure 4). These thermosets exhibit high $T_{g} \mathrm{~s}$ in the range of $127^{\circ} \mathrm{C}$ to $160^{\circ} \mathrm{C}$. The difference on $T_{g}$ of the BAPB/DGEBA ther- moset $\left(160^{\circ} \mathrm{C}\right)$ and the BHPB/DGEBA thermoset $\left(132^{\circ} \mathrm{C}\right)$ could be attributed to the reaction nature of amino and phenolic hydroxyl group towards oxirane group and molecular size of BHPB and BAPB $[8,9]$. On the other hand, the chain segment between the crosslink points of the BHPB/DGEBA thermoset is longer on the average than those of the BAPB/DGEBA thermoset.

For the ternary thermosets (B1-B4/DGEBA), $T_{g} \mathrm{~s}$ are in the range of $127-147^{\circ} \mathrm{C}$ and close to the $T_{g}$ of the BHPB/DGEBA thermoset $\left(132^{\circ} \mathrm{C}\right)$. Moreover, $T_{g}$ s of these ternary thermosets are not all in the range of $T_{g}$ of the BAPB/DGEBA thermoset and the BHPB/DGEBA thermoset as our original expectation. This reveals that BHPB with longer 
molecules may have more impact on the chain structure of the thermoset. Moreover, the non-sen-

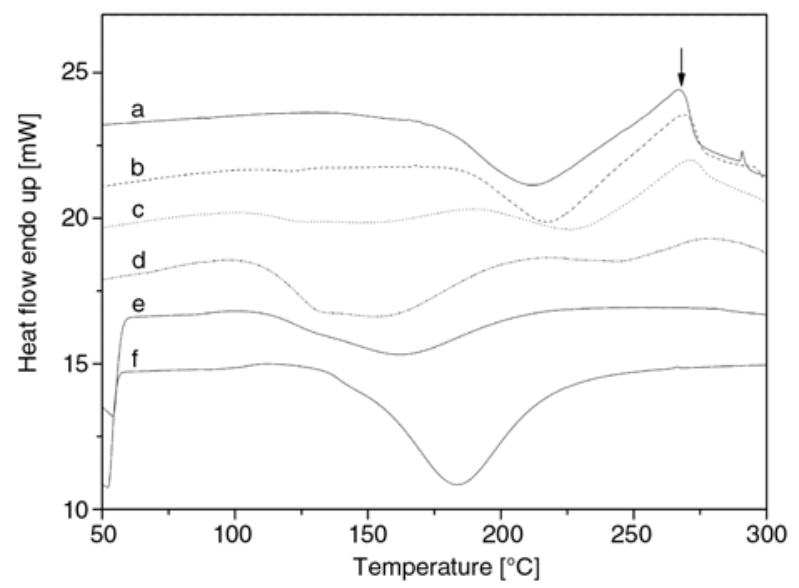

Figure 3. The DSC thermograms of DGEBA cured with a) BHPB; b) B1 (BHPB/BAPB = 2); c) B2 (BHPB/ $\mathrm{BAPB}=1.5)$, d) B3 (BHPB/BAPB = 0.33); e) B4 $(\mathrm{BHPB} / \mathrm{BAPB}=0.125)$ and $\mathrm{f}) \mathrm{BAPB}$ under $\mathrm{N}_{2}$ at a heating rate of $10^{\circ} \mathrm{C} / \mathrm{min}$

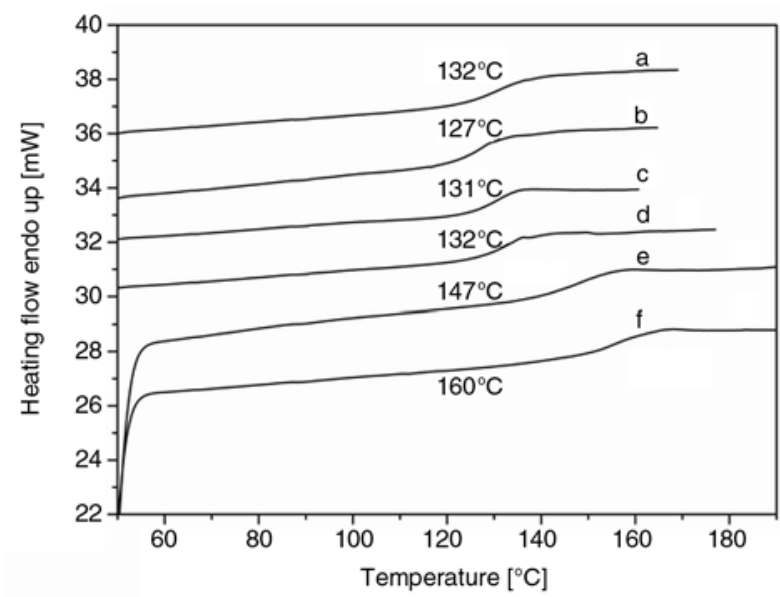

Figure 4. The $T_{g}$ of BHPB/BAPB/DGEBA thermosets from a second DSC trace under $\mathrm{N}_{2}$ at a heating rate of $20^{\circ} \mathrm{C} / \mathrm{min}$. a) BHPB/DGEBA; b) B1/ DGEBA; c) B2/DGEBA; d) B3/DGEBA; e) B4/ DGEBA and $f$ ) BAPB/DGEBA sitive relationship of $T_{g} \mathrm{~s}$ towards the networking structure of these thermosets might stem from the imperfect curing technique because all samples were cured under the same conditions, which may not be the optimum condition for the realization of their potential effect.

Although BAPB and BHPB contain flexible aromatic ether bond, they still exhibit relatively high $T_{g}$ s. So these epoxy thermosets containing azomethine moiety were potentially useful as advanced composite matrices because of high $T_{g}$ as well as excellent thermal stability, which will be discussed in detail in the following.

Figure 5 shows the TGA thermograms of the BHPB/BAPB/DGEBA thermosets. The thermal parameters $\left(T_{d, 5 \%}\right.$, and $\left.I P D T\right)$ of these ternary thermosets are listed in Table 1. It can be seen that the $T_{d, 5 \%}$ of these thermosets are all above $330^{\circ} \mathrm{C}$ and higher than that of DGEBA/dicyandiamide thermoset (about $290^{\circ} \mathrm{C}$ from our experiment). This

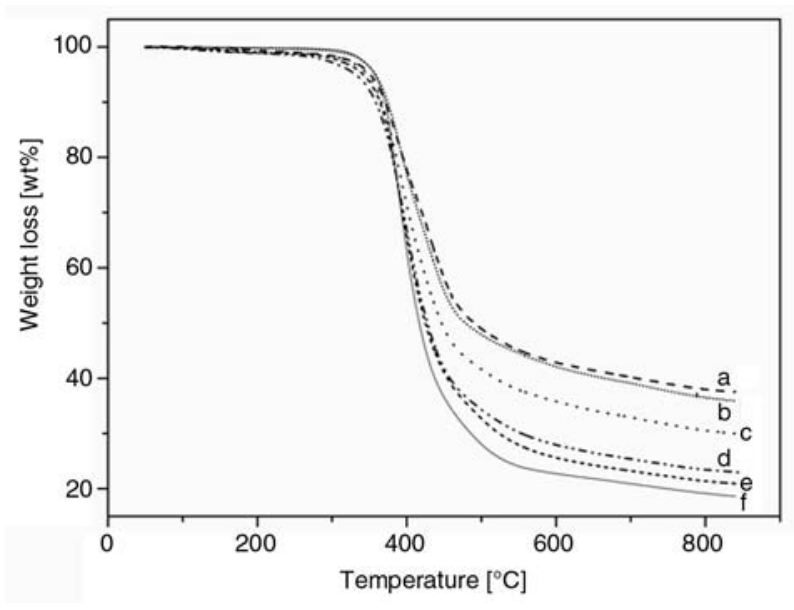

Figure 5. TGA thermograms of the ternary cured polymers, in $\mathrm{N}_{2}, 10^{\circ} \mathrm{C} / \mathrm{min}$. a) BHPB/DGEBA; b) B1/DGEBA; c) B2/DGEBA; d) B3/DGEBA; e) B4/DGEBA and f) BAPB/DGEBA

Table 1. Thermal stability parameters and $L O I$ values of the epoxy thermosets

\begin{tabular}{|l|c|c|c|c|c|c|c|}
\hline \multicolumn{1}{|c|}{ The epoxy thermosets } & $\mathbf{T}_{\mathbf{g}}\left[{ }^{\circ} \mathbf{C}\right]$ & $\mathbf{T}_{\mathbf{d}, \mathbf{5} \%}{ }^{\mathbf{1}}\left[{ }^{\circ} \mathbf{C}\right]$ & $\boldsymbol{Y}_{\boldsymbol{c}} \mathbf{\%}\left(\mathbf{8 5 0}{ }^{\circ} \mathbf{C}\right)$ & $\boldsymbol{A}^{*}$ & $\boldsymbol{K}^{*}$ & $\mathbf{I P D T}^{*}{ }^{\circ} \mathbf{C}^{\mathbf{2}}$ & $\mathbf{L O I}^{\mathbf{3}}$ \\
\hline BAPB/DGEBA & 160 & 357 & 18.61 & 0.574 & 1.347 & 662 & 26.0 \\
\hline B4/DGEBA & 146 & 331 & 22.94 & 0.595 & 1.466 & 743 & 29.5 \\
\hline B3/DGEBA & 130 & 350 & 27.70 & 0.634 & 1.734 & 919 & 31.0 \\
\hline B2/DGEBA & 131 & 347 & 29.82 & 0.638 & 1.759 & 948 & 32.9 \\
\hline B1/DGEBA & 127 & 357 & 35.90 & 0.681 & 2.138 & 1202 & - \\
\hline BHPB/DGEBA & 132 & 350 & 37.54 & 0.686 & 2.181 & 1230 & - \\
\hline
\end{tabular}

$15 \mathrm{wt} \%$ decomposition temperature $\left(T_{d, 5 \%}\right)$, indicates the apparent thermal stability of the epoxy thermosets;

${ }^{2}$ Integral procedure decomposition temperature (IPDT), proposed earlier by Doyle [13] can be discussed in a quantitative thermal analysis containing the char residue of the resulting thermosets at high temperature. From the TGA results, the IPDT is calculated by the following equation: $\operatorname{IPDT}\left({ }^{\circ} \mathrm{C}\right)=A^{*} K^{*}\left(T_{f}-T_{i}\right)+T_{i}$, where $A^{*}$ is the area ratio of total experimental curve divided by total TGA thermogram, $K^{*}$ is the coefficient of $A^{*}, T_{i}$ and $T_{f}$ are the initial and final experimental temperature respectively;

${ }^{3}$ the B1/DGEBA and BHPB/DGEBA thermosets were not cured to that extent that the $L O I$ measurement could be carried out. 


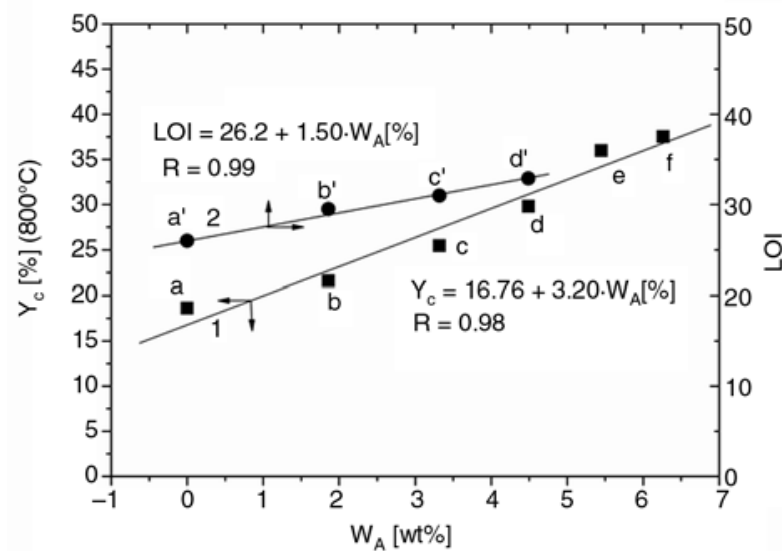

Figure 6. The relationship of char yield $\left(Y_{c}\right)$ and content of azomethine in the BAPB/BHPB/DGEBA thermosets. Diamine: bisphenol $=\left(\mathrm{a}, \mathrm{a}^{\prime}\right)$ 0:1; $\left(\mathrm{b}, \mathrm{b}^{\prime}\right)$ 2; (c, c') 1.5; (d, d') 0.33; (e) 0.125 and (f) $1: 0$

indicates that they all have excellent thermal stability and can meet the requirement of 'lead-free' copper clad laminate $\left(T_{d, 5 \%} \geq 330^{\circ} \mathrm{C}\right)$. The char yields $\left(Y_{c}, \mathrm{wt} \%\right)$ of these thermosets increase with increasing molar content of BHPB for the introduction of azomethine moiety which had been proved to facilitate formation of char residue during decomposition [10,11]. As shown in Figure 6, $Y_{c}$ increases with increasing $W_{A}\left(W_{A}\right.$, the weight percentage of azomethine in a sample) and exhibits good linearity $(R=0.98)$. According to Van Krevelen's theory [12], increase of the char formation will depress the flammability of the materials. Thus, it is rational to assess flame retardancy of these thermosets via char residue. The integral procedure decomposition temperatures (IPDT) of these thermosets increase dramatically with increasing molar content of BHPB from Table 1. This means that the inherent thermal stability of these thermosets was improved due to the introduction of azomethine moiety, which may be useful for improving the flame retardancy.

Limited oxygen index ( $L O I)$ measures the minimum oxygen concentration (in a flowing mixture of oxygen-nitrogen gas) required to support candlelike downward flame combustion [2]. A material with $L O I$ of 26 or higher is rated as a flame-retardant material. It is strongly dependent on the char residue for halogen-free polymers [12] and is suitable as a semi-qualitative indicator of the effectiveness of the flame retardants in the laboratory. Herein, we found that $L O I$ values (26.0-32.9) of these thermosets are beyond 26 and higher than that of the common epoxy thermoset (LOI of DGEBA/ dicyandiamide thermoset is about 19.8) and increase linearly with increasing the content of azomethine moiety (Figure 6, line 2). This shows that the resultant thermosets were endowed with flame retardancy due to the introduction of azomethine moiety.

\section{Conclusions}

In summary, a novel bisphenol BHPB, which contains azomethine moiety and flexible aromatic ether linkage, was synthesized and introduced into the curing system composed of diglycidyl ether of biphenyl A (DGEBA) and diamine. The resultant epoxy thermosets containing BHPB had high thermal stability and good flame retardancy, and can be potentially applied in the electrical/electronic industries, especially can be used as the environment friendly 'green' materials.

\section{References}

[1] Wang C. S., Shieh J. Y.: Synthesis and properties of epoxy resins containing 2-(6-oxid-6H-dibenz<c,e〉 $<1,2>$ oxaphosphorin-6-yl)1,4-benzenediol. Polymer, 39, 5819-5826 (1998).

[2] Lu S. Y., Hamerton I.: Recent Developments in the chemistry of halogen-free flame retardant polymers. Progress in Polymer Science, 27, 1661-1712 (2002).

[3] Zhang F., Zhang J., Wang Y. Modeling study on the combustion of intumescent fire-retardant polypropylene. Express Polymer Letters, 1, 157-165 (2007).

[4] Toldy N., Tóth P. A., Marosi G. C.: Synthesis of phosphorus-based flame retardant systems and their use in an epoxy resin. Polymer Degradation and Stability, 91, 585-592 (2006).

[5] Mormann W., Bröcher M., Schwarz P.: Mesogenic azomethine based diepoxides-monomers for the synthesis of 'liquid crystal' thermoset networks. Macromolecular Chemistry and Physics, 198, 3615-3626 (1997).

[6] Castell P., Serra A., Galia M., Giamberini M., Carfagna C.: Anisotropic thermosets from liquid-crystalline azomethynic epoxy resins and primary aromatic diamines. Journal of Polymer Science Part A: Polymer Chemistry, 41, 1-12 (2003).

[7] Mormann W., Bröcher M.: Liquid crystalline thermosets from triaromatic azomethine group containing diepoxides and 4,4'-methylenedianiline. Macromolecular Chemystry and Physics, 199, 1935-1938 (1998).

[8] Myers G. E., Dagon J. R.: Distribution of molecular weight and of branching in high molecular weight polymers from bisphenol a and epichlorohydrin. Journal of Polymer Science Part A: Polymer Chemistry, 2, 2631-2640 (1964). 
[9] Zhang X. H., Chen S., Min Y. Q., Qi G. R.: Synthesis of novel bisphenol containing phthalazinone and azomethine moieties and thermal properties of cured diamine/bisphenol/DGEBA polymers. Polymer, 47, 1785-1795 (2006).

[10] Mikroyannidis J. A.: Self-curing epoxy compounds. Journal of Applied Polymer Science, 41, 2613-2624 (1990).
[11] Palaninathan K., Singaravelu R., Pachagounder S.: Synthesis and characterization of thermotropic liquid crystalline poly(azomethine ether)s. Polymer, 45, 7895-7902 (2004).

[12] van Krevelen D. W.: Some basic aspects of flame resistance of polymeric materials. Polymer, 16, 615620 (1975).

[13] Doyle C. D.: Estimating thermal stability of experimental polymers by empirical thermogravimetric analysis. Analytical Chemistry, 33, 77-79 (1961). 